\title{
Internalizing Cognitive Bias: An Experiential Exercise for Teaching and Learning the Anchoring Effect
}

\author{
John C. Kleefeld \\ University of New Brunswick \\ Dionne Pohler \\ University of Toronto
}

\begin{abstract}
The ability to make good decisions is key to personal and professional success for students. In this case study, we outline a set of in-class exercises that we have used for students in business, law, human resources, and public policy to help them understand and internalize their susceptibility to cognitive errors. Specifically, we illustrate an experiential way to teach and learn the anchoring effect: a cognitive bias that causes decision-makers to rely too heavily on initial information when making subsequent judgments. We describe an anchoring exercise that can be easily adapted to various settings, and show its effectiveness in achieving the learning outcomes based on aggregated classroom data and our own experiences of student reactions. We show the robustness of the exercise to adaptation-including the various iterations of the exercise that have evolved over the course of our teaching-and highlight the challenges we encountered. We also discuss how the exercise can be used to encourage students to consider anchoring's ethical implications, as well as strategies to safeguard against being anchored.
\end{abstract}

Keywords: cognitive bias, heuristic, anchoring effect, simulated learning, debriefing

John C. Kleefeld is professor and dean of law at the University of New Brunswick. He previously taught at the University of Saskatchewan, where he received the Provost's Award for Outstanding Innovation in Learning and the Brightspace/STLHE Innovation Award in Teaching and Learning. Before that, he was at the University of British Columbia, where he taught Negotiation and directed the Legal Research \& Writing Program.

Email: john.kleefeld@unb.ca

Dionne Pohler is an assistant professor at the Centre for Industrial Relations and Human Resources at the University of Toronto. Her research is focused on the design and outcomes of different governance arrangements in organizations and the employment relationship, policy implementation, and economic and social development. Before her appointment at the University of Toronto, she taught at the University of Saskatchewan. She has received several university teaching awards.

Email: dionne.pohler@utoronto.ca

Brock Education Journal, 28(2), 2019 
As research-active faculty members who teach in professional schools and executive education programs, we are committed to closing the gap that students perceive between "academic research" and "practice." We often find it difficult, however, to translate research on complex empirical phenomena into something that is easier and more intuitive for students to understand, and (ideally) internalize. In the courses we have taught in law, business, industrial relations, public policy, public health, and human resources, it is common to include material about heuristics and cognitive biases - those traits that can enable quick decisions but also lead to perceptual distortions, inaccurate judgments, and illogical interpretations. Helping students recognize their cognitive biases is important because these predispositions are notoriously difficult to overcome, yet can have serious consequences (Cirka \& Corrigall, 2010).

One of the most prevalent and persistent cognitive biases, and one highly amenable to measurement, is the anchoring effect: the tendency to be overly influenced by the first piece of information offered in a scenario (e.g., a negotiation). We want professional students to understand how robust the anchoring effect is, even among experts who profess to be immune from it, and to consider the strategic and ethical aspects of being anchored or of anchoring another. Accordingly, we have used some simple exercises designed to teach the concept in a personal and visceral way and stimulate student reflection and class discussion. In this article, we report on the results of some of these efforts, and outline considerations for instructors who want to adapt the exercises for use in their classroom.

\section{The Anchoring Effect}

The anchoring effect is a cognitive bias in which information first learned about a subject (or, more generally, information learned at an early stage) affects future decision-making (Furnham \& Boo, 2011). We routinely make value judgments by referring to some prior information - such as a first offer price for a car, a sticker price for retail goods, the hourly wage earned by comparator workers, the prison sentence sought for a convicted offender, or the symptoms that a patient presents to a health professional in a medical consultation. How we "refer" to this information is the subject of much theoretical and empirical research. Do we start with the anchor and adjust the boundary of the range of plausible values ("anchor and adjustment") or do we activate latent information that is consistent with the anchor presented ("confirmatory search" or "selective accessibility")? Numerous empirical studies examine these and other mechanisms associated with the anchoring effect (Furnham \& Boo, 2011).

Kahneman, Slovic, and Tversky (1982) use an example of the anchoring effect, in which visitors at the San Francisco Exploratorium were asked to estimate the height of the tallest redwood. Half were asked the following questions in the order shown:

Is the height of the tallest redwood more or less than 1,200 feet?

What is your best guess about the height of the tallest redwood?

The other half were asked the following questions in the order shown:

Is the height of the tallest redwood more or less than 180 feet?

What is your best guess about the height of the tallest redwood?

The two groups had very different mean estimates of the tallest redwood's height. The mean for the first group was 844 feet; for the second, it was 282 feet. Even with the manipulations that may be adopted to test particular theoretical mechanisms and cognitive processes, many experimental anchoring studies follow a similar format for measuring the anchoring effect.

The anchoring effect is one of the most robust cognitive biases. Experts and non-experts alike are highly susceptible to it. In a well-known study conducted by Northcraft and Neal (1987), two

Brock Education Journal, 28(2), 2019 
groups - undergraduate business students and experienced realtors - were shown a house and given an information-rich package about its features, then asked to appraise its value. The only difference in the information provided to the groups was the list prices: some were anchored high; others, low. After making an appraisal, participants in each group were asked what factors influenced their decisions. The results? The estimates of both groups were significantly influenced by the anchored list price. The only notable difference was that the realtors, unlike the students, denied being influenced by the anchor.

The anchoring effect is even robust when participants are told that the "prior information" is randomly generated - and, remarkably, even when they take part in its (supposedly) random generation. In a study done by Englich, Mussweiler, and Strack (2006), 39 legal professionals, mostly judges, had to impose a sentence in a fictitious case concerning a woman who had stolen items from a store for the 12th time. Participants were first given a prosecutor's sentencing demand that was either high ( 9 months on probation) or low (3 months on probation). Instructions explained that this demand had been determined randomly, and did not represent any judicial expertise. Sentencing decisions for the shoplifting case varied between acquittal and 12 months on probation. Judges exposed to the high demand gave higher sentences (mean of 6.05 months) than those exposed to the low demand (mean of 4.00 months), a statistically significant difference. In another study discussed by the same researchers (Englich et al., 2006), 52 junior lawyers from different German courts who had acquired their first experiences as judges received the same materials and procedures as in the previous study, but instead of being told that the sentencing demand had been randomly chosen, they were told to determine the demand themselves by throwing a pair of dice. About half the participants received a pair of dice loaded so that they always showed 1 and 2; the other half received a pair of dice loaded so that they always showed 3 and 6 . After throwing the dice, participants were told to calculate the sum of the two dice and to fill it in as the prosecutor's sentencing demand in the questionnaire. In other words, they got the same anchors as in the first study-3 months and 9 months - but selfgenerated by what they thought was a random process. Sentencing decisions again varied substantially, ranging from 1 to 12 months on probation, with further analysis again revealing that the sentences were influenced by the random sentencing anchors. Judges exposed to the high anchor gave higher final sentences (mean of 7.81 months) than those exposed to a low anchor (mean of 5.28 months), with the difference in means being statistically significant.

\section{Learning Objectives}

The anchoring effect provides an effective way to teach students about cognitive biases because it is simple to demonstrate, highly robust to instructor idiosyncrasies, and easy to adapt to different contexts and learning objectives. Below we outline some learning objectives for our exercise that guided our approach to debriefing the exercise with our students.

1. Students will learn to recognize their cognitive limitations and how those limitations can affect their judgment.

2. Students will be able to identify potential strategies to reduce the effect of anchoring on their judgments and decisions.

3. Students will critically evaluate the ethical implications of using anchoring in bargaining strategies and tactics.

We most often used this exercise in the context of teaching negotiation, so the third learning objective may be less relevant for other instructors. However, instructors should feel comfortable 
in adapting the debrief and lecture (and even the quizzes themselves) to achieve their learning objectives and course outcomes.

\section{The Exercise}

We developed and administered three different anchoring exercises in the form of short classroom quizzes. Appendix A shows all adaptations of the exercise that we have used. The first "quiz" was adapted from materials used by Kleefeld (Folberg \& Golann, 2011, pp. 99-102); the second two we developed ourselves. The purpose of adapting the initial exercise was to make it more realistic and practically relevant for students from different backgrounds and to make it fit better with the specific learning objectives and content in particular disciplines. We also identified problems with using the original exercise, which we outline later. Generally, only one "quiz" should be used in any particular class. Each quiz posed two questions. The first was the anchoring question (which included either the high or low anchor - the high anchor is listed in brackets); the second question asked students for their best estimate of the actual answer.

\section{Step 1: Preparation}

We printed the two versions of the exercise (i.e., the high and low anchor) on a sheet of paper, cut it in half, and interwove the half-sheets to make a stack that alternated the high- and lowanchored versions. We also prepared slides for a lecture on judgment and decision-making errors (see Bazerman \& Moore, 2013 for one content source), as well as the debriefing and associated lecture to summarize key takeaways.

\section{Step 2: Exercise Instructions}

At the beginning of the class, or at some point that made sense in the context of the lesson plan, we introduced the exercise, but with very little information. The first author (Kleefeld) would typically use a statement like "we'll be doing a short warm-up exercise about decisionmaking under uncertainty" and the second author (Pohler) would say "I have a short quiz that I want you to take before we discuss today's content, and which we'll return to later" or tell the students that they would receive a pop quiz (not for credit) designed to test their knowledge of a particular subject area. Because anchoring studies usually involve a form of mild deception (comparator groups get different anchors for the same question without realizing it), we do not advise placing additional stress on students by suggesting the quiz will be for credit. We have not identified any problems with students taking the quiz less seriously as a result. We always revealed the deception in a debriefing session, which is critical for students to be able to internalize the learning: see Step 7 below. We instructed the students to fill in the answers on the half-sheet without consulting any other sources, discussing the quiz, or looking at their neighbours' answers.

\section{Step 3: Administering the Exercise}

We distributed the exercises, placing a quiz face down on the desk in front of each student. We then instructed the students to turn the quiz over, answer the questions, and place the quiz face down on the desk upon finishing it. We suggest giving students just a minute or two to complete the exercise. We then collected all the quizzes. While the enrolment in each of our 
classes varied from 10-45 students, the exercise usually took no more than 10 minutes to complete, from the introduction of the exercise to the collection of all completed quizzes

While we followed similar procedures in administering the exercises, style differences and variations in class format, class size, and disciplines required slight adaptations to the approach used. Below, we discuss what challenges these inconsistencies may introduce, and highlight what we think are best practices for anyone who wants to reproduce or adapt the exercises for particular classroom settings.

\section{Step 4: Take a Break to Tally the Results}

It may be useful to conduct this exercise before a break; however, it is also possible to simply put the quizzes aside and tell the students that you will return to the results after a scheduled break. We have found it ideal to conduct the exercise prior to a lecture that surveys psychological aspects of negotiation or decision-making, including cognitive biases - but to then reveal the results after covering this material. It is most beneficial to reveal the results within the context of the same class in which the quiz is administered. Thus, usually during a break, we would enter the data into a pre-configured Excel spreadsheet that calculated the mean estimates, for example, for the population of Iran or the average minimum wage for each of the low- and high-anchored groups. Where there is no class break or time is otherwise limited, it may be possible to enlist the help of an assistant, co-instructor, or volunteer student from another class to do the data entry while the main instructor is teaching.

\section{Step 5: Reveal the Results}

At the point in the lecture where we cover the anchoring effect, we would reveal the intent of the quiz and the corresponding results. We have used variations of writing the two columns of estimates on the whiteboard or showing them on a PowerPoint slide. Regardless of which approach is used to reveal the results, we have found it most useful to write the individual responses in columns, along with the mean differences between the two groups and an indication of whether the difference is statistically significant, rather than simply reporting the aggregated means. When students can see their own estimates in the listing, it helps them internalize the results in a visceral way. When we have simply reported the aggregate means, the exercise has still been effective, but appears to have less of an impact on individual students. We neither collected the student names nor wrote them beside the numbers, but the students easily recognized their estimates.

When the students see the values they submit via the quizzes, we have observed them to experience two levels of mild shock: one at seeing the difference in the mean estimates; the other at seeing how much they were anchored themselves. At this point, there is usually quite a "buzz" in the classroom. We have found that the combination of the mild deception and revealing the results in this manner leads to a deeper engagement with the anchoring effect than the lecture itself.

\section{Step 6: Debriefing Session}

Debriefing is crucial in any kind of pedagogical role play or simulation, and more so when deception is part of the pedagogy (Taras \& Steel, 2007) even if, as in this case, the deception is very mild. The purpose of the debriefing is to give students an opportunity to take time to 
consider what just happened and how it relates to what they have been learning - and to thereby become reflective practitioners. In "Debriefing the Debrief," Deason et al. (2013) explain that debriefing should:

... make the experiences of the learning activity come alive[,] connect those experiences to the content of the course[,] assist participants in putting theory into practice and ... make the process feel personal and real, and increase the likelihood that the students will remember and use their new learning. It should help students develop the habit of reflecting on their practice. (p. 302)

Immediate debriefing of in-class exercises requires an understanding of the variety of outcomes that may occur. It also requires some flexibility on the instructor's part to be able to adapt the debriefing in real time so as to be relevant for the emergent discussion and course learning objectives.

We generally had students discuss the results openly before summarizing key lessons, and then guided the discussion to where we thought it was important to draw specific conclusions. Depending on the nature of the course and the instructor's comfort level in facilitating class discussion, the debriefing can also be controlled by posing specific questions, or by providing a lecture to draw out key lessons. We aimed for a mix of open student reactions, and an instructorled follow-up discussion.

After noting everyone's susceptibility to cognitive biases even if they are aware of them, students often find it most intriguing to engage in a discussion about the ethics of using anchoring as a bargaining tactic. A student will inevitably muse that this may be one reason why first offers often appear so unrealistic. This observation can result in a discussion about what implications different strategies might have on building and maintaining trust, particularly in the context of long-term relationships (e.g., union-management negotiations) if the other party understands they are being anchored. For some students, this may be the first time they have thought about this.

We encourage following up the debriefing with a short discussion on how students can develop strategies, structures, and processes to reduce the anchoring effect on themselves. For example, in medicine, research has shown that about $75 \%$ of diagnostic errors have a cognitive component, including anchoring and confirmation bias (the tendency to selectively seek information that supports initial impressions); strategies to combat such effects include case discussions and actively seeking information that could lead away from the initial impression (Etchells, 2015). Sometimes we use the debriefing as a starting point to discuss additional cognitive biases, such as framing, escalation of commitment, availability bias, and halo effects, depending on the learning objectives for a given course.

\section{Outcomes}

\section{Assessment of Learning Objectives}

Most of our assessment of whether we achieved our particular set of learning objectives have been drawn from our own experiences in comparing the use of this exercise with approaches that were primarily lecture-based. In particular, we observed that students could immediately recognize and internalize their cognitive limitations, given their active participation in the exercise. Moreover, students appeared more animated in group discussions around possible strategies to reduce the effect of anchoring on their behaviours. The exercise made the reality of being anchored much more personal for students than a lecture alone ever could. For instance, in 
an extended email negotiation assignment done by law students after they had taken part in one of the anchoring exercises discussed in this article, students experienced anchoring as having both ethical and substantive consequences; as affecting the distribution of power and resources between parties; and as having practical (sometimes undesired) cause-and-effect consequences for the relationship that the parties were trying to build (Kleefeld \& Keet, 2011).

\section{Robustness of the Exercise Across Settings}

We encourage instructors to adapt our exercise to suit their learning objectives and disciplinary context, and toward this end offer some evidence of the robustness of our exercises to adaptation across different classroom settings. From 2010-2013, Kleefeld conducted in-class anchoring exercises in six sections of Law 430: Negotiation. In 2013, both authors met to discuss developing this exercise as a teaching tool in different settings. From 2013-2016, we conducted in-class exercises in several courses at the undergraduate and graduate levels at the University of Saskatchewan in business, public policy, human resources, industrial relations, and law. In total, we conducted 19 teaching exercises from 2010-2016. All exercises were developed and administered by one of us except for four administered in 2014-2015 by another colleague who taught Law 430 while Kleefeld was on leave. Kleefeld trained this colleague in administering the exercise.

Five exercises were administered outside a university course setting: one as part of a scholarship-of-teaching-and-learning (SoTL) presentation for faculty members at the University of Saskatchewan delivered by both of us, and four by Pohler as part of a professional certificate program offered through the University of Saskatchewan. The participants in these five exercises could be considered "experts." The anchoring exercises were in subject areas for which the individuals would have had greater knowledge (faculty salaries and minimum wages) than students in undergraduate and graduate courses due to their education and professional work experience. In total, 378 individuals took part in these exercises over 7 years in 19 classroom settings. A summary of participants in the anchoring exercises for each type of quiz and course is shown in Table 1.

Table 1

Participants in Anchoring Exercises, University of Saskatchewan, 2010-2016

\begin{tabular}{lccccccc}
\hline & \multicolumn{5}{c}{ Class } & \\
\cline { 2 - 6 } Quiz & COMM489 & POL815 & LAW430 & MBA & LMR* & SoTL* & Total \\
\hline Global knowledge & \multirow{2}{*}{23} & 23 & 37 & 34 & 104 & & 110 \\
Minimum wage & 23 & & 40 & & & 7 & 47 \\
Faculty salary & & & & & & & \\
\hline
\end{tabular}

*expert

COMM489: undergraduate HR course-University of Saskatchewan

POL815: graduate HR/labour policy course-University of Saskatchewan

LAW430: negotiation course-University of Saskatchewan

MBA: master of business administration strategic HR course-University of Saskatchewan

LMR: labour-management relations certificate module-University of Saskatchewan

SoTL: scholarship of teaching and learning-faculty symposium

To evaluate the consistency and overall effectiveness of this exercise in highlighting the anchoring effect, we compiled all data from the exercises administered from 2010-2016, as well 
as from the classes of the other faculty member mentioned above. We conducted three types of analyses. The first was calculation of the anchoring index for each exercise, which is the ratio of the difference between the mean of the estimates for each group and the difference between the anchor values. This is a standard measure for the anchoring effect and has the advantage of being unit-free. For example, the anchoring index for the results from the San Francisco Exploratorium exercise mentioned earlier (Kahneman et al., 1982) would be $(844 \mathrm{ft}-282 \mathrm{ft}) \div(1200 \mathrm{ft}-180$ $\mathrm{ft})=0.55($ or $55 \%)$. An index of 0 indicates no anchoring effect at all; as the index approaches 1 , it indicates an extreme anchoring effect. The second calculation was the $p$-values for the difference between the mean group estimates for the high and low anchors for each exercise using a one-tailed $t$-test, assuming unequal variances. The third was a regression analysis that tested whether expertise could mitigate the anchoring effect. The results from the first two analyses are shown in Table 2; the regression results are shown in Table 3.

Table 2

Anchoring Indices and Significance Tests for Classroom Exercises

\begin{tabular}{lcccccc}
\hline Exercise & Quiz & High mean & Low mean & $\begin{array}{c}\text { Absolute } \\
\text { difference }\end{array}$ & $P$-value & $\begin{array}{c}\text { Anchoring } \\
\text { index }\end{array}$ \\
\hline 1 & global & $63,625,026$ & $22,425,000$ & $41,200,026$ & 0.032 & 0.22 \\
2 & global & $116,600,000$ & $28,400,000$ & $88,200,000$ & 0.012 & 0.47 \\
3 & global & $79,727,273$ & $6,742,490$ & $72,984,783$ & 0.001 & 0.39 \\
4 & global & $83,368,333$ & $43,833,333$ & $39,535,000$ & 0.072 & 0.21 \\
5 & global & $176,857,143$ & $10,347,303$ & $166,509,840$ & 0.000 & 0.90 \\
6 & global & $497,031,519$ & $19,675,000$ & $477,356,519$ & 0.068 & 2.57 \\
$7^{*}$ & minwage & $\$ 11.22$ & $\$ 9.21$ & $\$ 2.01$ & 0.001 & 0.27 \\
8 & minwage & $\$ 11.58$ & $\$ 9.78$ & $\$ 1.80$ & 0.023 & 0.24 \\
9 & minwage & $\$ 12.17$ & $\$ 8.64$ & $\$ 3.53$ & 0.000 & 0.47 \\
10 & minwage & $\$ 10.46$ & $\$ 7.78$ & $\$ 2.68$ & 0.008 & 0.36 \\
11 & minwage & $\$ 11.68$ & $\$ 9.38$ & $\$ 2.30$ & 0.002 & 0.31 \\
12 & minwage & $\$ 11.58$ & $\$ 9.00$ & $\$ 2.58$ & 0.001 & 0.34 \\
$13 *$ & minwage & $\$ 10.33$ & $\$ 9.56$ & $\$ 0.77$ & 0.105 & 0.10 \\
$14 *$ & facsalary & $\$ 112,500$ & $\$ 96,333$ & $\$ 16,167$ & 0.123 & 0.20 \\
15 & minwage & $\$ 11.00$ & $\$ 9.13$ & $\$ 1.87$ & 0.046 & 0.25 \\
16 & facsalary & $\$ 119,444$ & $\$ 90,300$ & $\$ 29,144$ & 0.009 & 0.36 \\
17 & facsalary & $\$ 117,700$ & $\$ 82,204$ & $\$ 35,496$ & 0.007 & 0.44 \\
$18^{*}$ & minwage & $\$ 11.63$ & $\$ 9.66$ & $\$ 1.97$ & 0.002 & 0.26 \\
$19 *$ & minwage & $\$ 11.06$ & $\$ 9.32$ & $\$ 1.74$ & 0.005 & 0.23 \\
\hline
\end{tabular}

*expert

We note a few things about Table 2. First, except for four exercises (Exercises 4, 6, 13, and $14)$, there is a statistically significant anchoring effect at conventional levels $(p<0.05)$ across every other exercise we conducted; that is, there is only a 5\% chance (or less) that the difference between the means of the high- and low-anchored groups was due to random error. Exercise 6 also achieves statistical significance $(p<0.01)$ when two outliers are removed. Exercise $14(n=7)$ would likely have achieved statistical significance with a larger sample since the absolute 
difference between the group means was relatively large. Second, the anchoring index varies widely, from $10 \%$ to $257 \%$. However, if the two data outliers are removed for Exercise 6 , the anchoring index reduces to $55 \%$, and the anchoring indices range from $10 \%$ to $90 \%$. Third, the average anchoring effect appears to be the smallest for the minimum wage quiz (28\%), next highest for the faculty salary quiz (34\%), and largest for the global knowledge quiz: $46 \%$ or $79 \%$, depending on whether the outliers from Exercise 6 are removed. The numbers in Table 2 are rounded and may differ slightly from the calculations reported here. Finally, on average, the anchoring indices appear to be lower for the expert groups than the regular students, though we examine this issue in more detail below.

One factor affecting the results is the relative extremity of the anchors. In the global population quiz, the high anchor of 190 million was almost 48 times that of the low anchor of 4 million, so it is not surprising that the anchoring effect was quite large in this exercise. In contrast, the high anchors in both the minimum wage quiz and the faculty salary quiz were, by design, just twice that of the low anchors $(\$ 15.00$ per hour $\$ 77.50$ per hour and $\$ 160,000$ per year $\$ 80,000$ per year). Yet even in these exercises, the differences in the means are striking. In the minimum wage quiz, the average difference is $\$ 2.13$. In the context of a range of $\$ 7.50$ to $\$ 15.00$ per hour, a difference of $\$ 2.13$ per hour is very large; in companies depending on minimum-wage labour, it could mean the difference between profit and loss. Similarly, in the average faculty salary quiz, the average of the differences in the mean estimates is $\$ 26,936$, which is also large in the context of the given range of $\$ 80,000$ to $\$ 160,000$ annually.

While the anchoring index appears to be affected by the type of quiz administered, the quizzes were neither balanced nor randomly assigned across other variables that could also affect the results (e.g., instructor and type of class). We also did not include an unanchored control group. Thus, our design cannot determine if the quizzes caused these differences. It may be the general level of knowledge of each quiz topic, the size of the numbers used, or something else. In future, it may be a good practice to include an unanchored baseline group to compare with the results of those who were anchored. Because our intention was not to test specific theories or research hypotheses, other studies would have to be designed to determine what mechanism leads to divergence across type of quiz or to answer specific research questions. In using the tool as a classroom learning exercise, we note that the type of quiz may have an impact on the extent of the anchoring effect observed. Moreover, the global knowledge population quiz of Iran appears to be more susceptible to response outliers.

The anchoring indices in Table 2 suggest that there may have also been an "expert" effect. For instance, the anchoring indices for the "expert" student respondents on both the minimum wage and faculty salary quizzes were lower on average than when the non-expert students took the same quizzes, and two of the expert exercises did not achieve statistically significant differences between the means of the anchored groups. Because it is difficult to compare descriptive results across exercises, we also conducted regression analyses at the individual level to determine whether experts may have been less susceptible to the anchoring effect than others. We only conducted this analysis on the responses for the minimum wage quiz, as we did not have experts complete the global knowledge quiz, and we have only a small number of responses to the faculty salary quiz.

The results of the ordinary least squares (OLS) regression analyses are shown in Table 3 . The dependent variable, RESPONSE, is the participant's estimate, ANCHOR is a dummy variable (high=1, low=0), EXPERT is a dummy variable (expert=1, non-expert=0), and INTERACTION is the product between the latter two variables. As expected, when participants received the high 
anchor on the minimum wage quiz, they responded on average with an estimate of $\$ 2.09$ more than when they received the low anchor. It does not appear that experts responded differently than students (i.e., the expert coefficient was insignificant). We ran a model with the interaction between ANCHOR and EXPERT, but this coefficient was also not significant. These results adjust the standard errors to take into account the clustered nature of the experiments. Overall, the results are consistent with research that has found experts to be as susceptible to anchoring as non-experts.

Table 3

OLS Regression Results for Minimum Wage Quiz

\begin{tabular}{lcclcc}
\hline & \multicolumn{2}{c}{ Main effects model } & & \multicolumn{2}{c}{ Interaction model } \\
\cline { 2 - 3 } \cline { 5 - 6 } Response & Coefficient & Std. err. & & Coefficient & Std. err \\
\hline Anchor & 2.09 & $(0.23)^{* * *}$ & & 2.44 & $(0.33)^{* * *}$ \\
Expert & -0.04 & $(0.25)$ & & 0.32 & $(0.33)$ \\
Interaction & & & & -0.73 & $(0.40)$ \\
Constant & 9.27 & $(0.26)^{* * *}$ & & 9.10 & $(0.32)^{* * *}$ \\
\hline R-squared & \multicolumn{2}{c}{0.25} & & \multicolumn{2}{c}{0.26} \\
N & \multicolumn{2}{c}{221} & & \multicolumn{2}{c}{221} \\
\hline
\end{tabular}

$* p<0.05, * * p<0.01, * * * p<0.001$

\section{Challenges, Limitations, and Best Practices}

As shown in the results of administering these exercises over 7 years, 19 classes, and 378 students, we almost always obtained a robust anchoring effect, though its size varied with the context. Instructors must be aware of how contextual factors may affect the results, so they can adapt and respond accordingly in the debrief.

One contextual factor, as discussed, was the anchor values. Other things being equal, more extreme anchor values appear to create larger anchoring effects. Another contextual factor may have been the extent of prior knowledge on the part of quiz participants. During the period in which the global population quiz was administered, the population of Iran averaged 75.7 million, growing from 74.3 million in 2010 to 77.2 million in 2013 (The World Bank, 2017). For a student from Iran or a second-generation Canadian with Iranian parents, prior knowledge of this figure, or an estimate of it, already existed. Kleefeld sometimes had such students, and when he would ask in the debriefing, "Does anyone know the actual population of Iran?" they would usually be able to give a close approximation-e.g., "around 70 million" (or if no one knew, he would ask someone to look it up on the Internet and tell the class).

In fact, these two contextual factors can be interrelated. If an anchor value is extreme, a person with prior knowledge will know it is. And yet studies show that even in the face of this knowledge, or presumed knowledge, participants still experience the anchoring effect. Two psychologists showed this in a study (Strack \& Mussweiler, 1997) in which they asked whether Mahatma Gandhi died before or after age 9, or before or after age 140. For anyone with a modicum of worldly knowledge and common sense, neither of these anchors could be correct, yet the two groups still guessed significantly differently (mean age of 50 versus mean age of 67; actual age of 78).

Brock Education Journal, 28(2), 2019 
In the debriefing, some students objected that the population quiz asked a question that most of them would be unable to answer and that this was one of the reasons they were so heavily influenced by the anchors. It was partly to eliminate this objection that we developed the two subsequent exercises. And it was partly to address the objection that "we were anchored because the differences were so extreme" that we capped the high:low anchor ratio to $2: 1$ in the minimum wage and faculty salary exercises. Thus not only was the subject matter closer to the interests and knowledge of those being quizzed, but the anchor points were much less extreme than in the population quiz. Furthermore, in the minimum wage exercise, the students could be expected to have had direct experience of the minimum wage in the provinces in which they had lived or worked, even though we would not characterize most of them as "experts." Again, we revealed the actual average unweighted minimum wage, calculated from Statistics Canada (2017) data: $\$ 10.22$ in January 2014 (it would become $\$ 11.26$ by January 2017). We were as surprised as the students at the extent of the anchoring effect and the difficulty of mitigating it even with worldly experience. Some students would acknowledge in the debriefing that they knew they were being anchored and that they had tried to compensate for this, yet were still anchored by the number in the initial question.

Even though we have had much success with using these in-class exercises, it is possible that they may not yield any anchoring effect, or even more unexpectedly, yield the opposite effect. If so, instructors may wish to have the usual kind of results on hand to show to students. In such a case, we also suggest engaging in a discussion about why the anchoring effect may not have been observed in this particular class. This could result from any number of factors, some of which relate to context, as discussed above. We also encourage instructors to highlight any outliers and to show the students the analysis both with and without them. For instance, in the global knowledge quiz, students would sometimes inadvertently add an extra zero to their answer.

The authors have found these exercises only slightly more difficult to conduct in larger classes. For classes over 30, we recommend having a teaching assistant, colleague, or former student help in administering an exercise. Classes should also not be too small, so that the anchoring effect may be observed. We recommend caution in adopting these exercises in classes with less than 10 students; there will probably not be enough data to generate a statistically meaningful difference in low- and high-anchored means. In smaller classes, instructors may choose to report these results, or the results of another anchoring study, though we see that approach as less effective in imparting the desired lesson-namely, that we are all susceptible to the anchoring effect.

There may be students who will have done this or a similar exercise and will thus have learned of its intention. This risk can be mitigated by the way the instructor introduces the exercise. However, as research has shown, and in our classroom experience, even though students may have done similar exercises before and know of the anchor, they are still susceptible. Indeed, for some of our classes, the pre-assigned readings for the day included an explanation of anchoring and results of anchoring studies.

\section{Ethics of Using Deception as a Teaching Pedagogy}

Do our exercises raise issues about the ethics of using deception as a teaching pedagogy? While the deception is minor and likely poses virtually no risk for either student or instructor, there is always potential for in-class deception to reduce trust. Neither author has experienced

Brock Education Journal, 28(2), 2019 
any serious issues or student concerns with the use of these exercises. One author found it useful to directly incorporate a discussion into the debriefing about the ethics of using deception as a teaching pedagogy, whether the students raised the issue or not. Instructors could also encourage students to comment on their experience of the exercise using end-of-year teaching evaluations if they prefer to remain anonymous, rather than raising the issue in class. In our experience, the benefits of using this mild form of deception have far outweighed the potential costs, at least for these exercises. However, the use of deception makes it important for the instructor to consider the relationship he or she has with the students, and whether the exercise respects the psychological contract that has been created in the class. It is also for this reason that a careful debriefing should be considered a basic requirement for administering these types of exercises.

\section{Conclusion}

Experiential learning exercises are effective ways to narrow the research-practice gap in classroom settings. We have proposed a type of classroom exercise that we believe is effective in teaching students about cognitive bias. We hope that other educators will find these exercises and strategies useful for teaching about the anchoring effect in their classrooms and that they will find ways to adapt our ideas to illustrate other cognitive biases, of which there are many. We also hope that these lessons are embedded in our students' minds as they progress in both their professional and personal lives. Future evaluations of the efficacy of this approach may do well to follow up with students later in their careers to determine whether they remember the particular exercise that they engaged in, and how it may have affected them.

Brock Education Journal, 28(2), 2019 


\section{Acknowledgments}

We thank our students - they taught us as much as we have taught them. We are also grateful to the University of Saskatchewan, including the Gwenna Moss Centre for Teaching Effectiveness, for supporting us in our interest in classroom innovations. We also thank Professor Michaela Keet (College of Law, University of Saskatchewan) for conducting our anchoring exercise in her classes, and for sharing the data with us. Finally, we thank Professor and former Dean Daphne Taras (Edwards School of Business, University of Saskatchewannow Dean, Ted Rogers School of Management, Ryerson University) for encouraging us to share the results of several years of classroom experiments with a wider audience.

Brock Education Journal, 28(2), 2019 


\section{References}

Bazerman, M., \& Moore, D. (2013). Judgment in managerial decision making (8th ed.). New York, NY: Wiley.

Cirka, C. C., \& Corrigall, E. A. (2010). Expanding possibilities through metaphor: Breaking biases to improve crisis management. Journal of Management Education, 34(2), 303-323. doi:10.1177/1052562909336912

Deason, E. E., Efron, Y., Howell, R. W., Kaufman, S., Lee, J., \& Press, S. (2013). Debriefing the debrief. In C. Honeyman, J. Coben, \& A. Wei-Min Lee (Eds.), Educating negotiators for a connected world (pp. 301-332). Saint Paul, MN: DRI Press.

Englich, B., Mussweiler, T., \& Strack, F. (2006). Playing dice with criminal sentences: The influence of irrelevant anchors on experts' judicial decision making. Personality and Sociology Bulletin, 32(2), 188-200. doi:10.1177/0146167205282152

Etchells, E. (2015, June). Anchoring bias with critical implications. PSNet. Retrieved from https://psnet.ahrq.gov/webmm/case/350/anchoring-bias-with-critical-implications

Folberg, J., \& Golann, D. (2011). Lawyer negotiation: Theory, practice and law (2nd ed.). New York, NY: Aspen Publishers.

Furnham, A., \& Boo, H. C. (2011). A literature review of the anchoring effect. The Journal of Socio-Economics, 40(1), 35-42. doi:10.1016/j.socec.2010.10.008

Kahneman, D., Slovic, P., \& Tversky, A. (Eds.). (1982). Judgment under uncertainty: Heuristics and biases. Cambridge, UK: Cambridge University Press.

Kleefeld, J., \& Keet, M. (2011). Getting real: Enhancing the acquisition of negotiation skills through a simulated email transaction. Journal of Arbitration and Mediation, 2, 23-52. Retrieved from https://papers.ssrn.com/sol3/papers.cfm?abstract_id=1934479

Northcraft, G. B., \& Neal, M. A. (1987). Experts, amateurs, and real estate: An anchoring-andadjustment perspective on property pricing decisions. Organizational Behavior and Human Decision Processes, 39(1), 84-97. doi:10.1016/0749-5978(87)90046-X

Statistics Canada. (2017). Current and forthcoming minimum hourly wage rates for experienced adult workers in Canada. Retrieved from http://srv116.services.gc.ca/dimt-wid/sm$\mathrm{mw} / \mathrm{rpt}$ 1.aspx?lang=eng

Strack, F., \& Mussweiler, T. (1997). Explaining the enigmatic anchoring effect: Mechanisms of selective accessibility. Journal of Personality and Social Psychology, 73(3), 437-446. doi:10.1037/0022-3514.73.3.437

Taras, D., \& Steel, P. (2007). We provoked business students to unionize: Using deception to prove an IR point. British Journal of Industrial Relations, 45(1), 179-198. doi:10.1111/j.1467-8543.2007.00607.x

The World Bank. (2017). Population, total: Iran, Islamic Rep. 1960-2017. Retrieved from http://data.worldbank.org/indicator/SP.POP.TOTL?cid=GPD_1\&locations=IR

Brock Education Journal, 28(2), 2019 


\section{Appendix A}

\section{Global Knowledge Quiz}

1. Is the population of Iran more or less than 4,000,000 $(190,000,000)$ people?

Circle the correct answer:

a. More than 4,000,000 $(190,000,000)$

b. Less than $4,000,000(190,000,000)$

2. What is the population of Iran?

Write down your best estimate:

\section{Minimum Wage Quiz}

1. Is the unweighted average minimum wage across the provinces and territories [of Canada] more or less than $\$ 7.50$ (\$15.00) /hour?

Circle the correct answer:

a. More than $\$ 7.50(\$ 15.00) /$ hour

b. Less than $\$ 7.50(\$ 15.00) /$ hour

2. What is the unweighted average minimum hourly wage across the provinces and territories?

Write down your best estimate:

\section{Faculty Salaries Quiz}

1. Is the current yearly average salary for a full-time Canadian university professor more or less than $\$ 80,000(\$ 160,000)$ ?

Circle the correct answer:

a. More than $\$ 80,000(\$ 160,000) /$ year

b. Less than $\$ 80,000(\$ 160,000) /$ year

2. What is the current yearly average salary for a full-time Canadian university professor? Write down your best estimate: 